\title{
EVALUATION OF THE ROLE OF MUTANT IDH1, EGFR AND BCL2 PROTEIN EXPRESSION IN DISTINGUISHING DIFFUSE ASTROCYTOMA FROM ASTROCYTOSIS
}

\author{
Fouad EM, Prof. Dr. Saba E.E.I., Prof. Dr. Rashed M.E.H. Prof.Dr. Abdelwahab R.M. \\ Pathology department, Faculty of Medicine, Zagazig University
}

\begin{abstract}
Background: Gliomas are the most relevant primary brain tumors. Diffuse astrocytomas are the most common type of gliomas .The distinction of diffuse infiltrating astrocytoma from reactive astrocytosis is one of the most difficult differential diagnosis especially with small biopsies (e.g. stereotactic biopsies). Several markers were used for this differentiation such as glial fibrillary acidic protein, ki67 and p53; but each of them has problems with sensitivity and specificity. The immunohistochemical markers such as mutant isocitrate dehydrogenase 1 (IDH1), EGFR and Bcl2 may be useful in this differentiation.

Aim: This study was conducted to clarify the value of IDH1, EGFR and Bcl2 protein expression in making a clear distinction between diffuse infiltrating astrocytomas and morphologically similar reactive astrocytosis.

Methods: Fifty representative cases; 30 cases WHO grade II diffuse astrocytomas and 20 cases of reactive conditions were examined immunohistochemically using antibodies against mutant IDH1, EGFR and Bcl2.

Results: IDH1 was positive in $60 \%$ of astrocytomas cases but it was totally absent in reactive astrocytosis. EGFR was expressed in $50 \%$ of astrocytomas; but it was not expressed in reactive astrocytosis. Bcl2 expression was detected in $56.7 \%$ of astrocytomas cases. However; it was expressed also in $75 \%$ of reactive astrocytosis cases.

Conclusion: IDH1, EGFR were the most useful IHC markers in the discrimination of low grade diffuse astrocytomas WHO II from reactive astrocytosis; particularly in small biopsies. Bcl2 play no role in this differentiation

Key words: Astrocytoma, Astrocytosis, IDH1, EGFR, BCL2
\end{abstract}

\section{INTRODUCTION}

P rimary brain tumors representing about $1.4 \%$ of all cancers and $2.3 \%$ of all cancer related deaths. The worldwide incidence rate of primary brain tumors per 100000 person-years; ranged from5.8 for males in developed countries (4.1 for females) to 3.0 for males in less developed countries (2.1 for females) [1].

In Egypt; it was represented per 100000 personyears:5.4 for males and 3.7 for females [2]. Gliomas are the most relevant primary brain tumors; representing about $81.7 \%$. Diffuse astro-cytomas are the most common type of gliomas representing about $67.6 \%$ of gliomas [3].

Diffuse astrocytoma WHO grade II is an infiltrating tumor; the major differential diagnosis is reactive astrocytosis in the setting of a nonneoplastic process particularly on small biopsies. That is a critically important one; because if the pathologist cannot make the decision with confidence, proper treatment is delayed, and there likely is a need for a second biopsy. The most commonly used markers to differentiate astrocytoma from astro-cytosis are glial fibrillary acid protein, proliferation markers (e.g. Ki-67) and TP53 [4].

Glial fibrillary acid protein (GFAP) is used to highlight astrocyte distribution, architecture and types of processes. However, these stains do not always permit accurate diagnosis, particularly because reactive astrocytosis occurs in astrocytomas often at the infiltrating edges [5].

Ki67 (MIB-1) proliferating labeling index is around $1 \%$ in reactive astrocytes. However, it can be high in some reactive conditions as progr- essive multifocal leukoenceph-alopathy (13\%), also there is inter observer variability among $\mathrm{Ki}$ 67 inter-pretation and different cut off values for what constitutes a "high" Ki-67 index. So it is not definitive for the differential diagnosis [6].

TP53 is used to differentiate reactive from neoplastic astrocytic lesions but it is an inaccurate marker. It is neither sensitive nor specific since it may show light labeling of non-neoplastic cells; not all TP53 gene mutations result in immunohistochemically detect-able TP 53; and some reactive conditions as progressive multifocal leukoenceph-alopathy may show strong positivity for TP 53 [7].

Two isoforms of IDH (IDH1 and IDH2) are mutated in high proportions in glioma. IDH1 mutations have not been found in reactive astrocytic conditions and seem to be specific to gliomas in more than $70 \%$. The vast majority about $90 \%$ of IDH1 mutation involve amino acid substitution at residue 132 (R132H) with CGT-CAT transition causing a specific amino acid change from arginine to histidine (R132H) [8].

Tumors with IDH1 mutation had better outcome than those with wild type.Epidermal growth factor receptor (EGFR) is a $170-\mathrm{kDa}$ trans-membrane tyrosine kinase receptor expressed in a variety of normal and malignant cells. Several ligands including EGF and TGF alpha may activate EGFR. EGFR activation is involved in several mecha-nisms including cell survival, prolifer-ation and migration. Its expression is lower in reactive gliosis than in the low and high grade astrocytomas [9]. 
The anti-apoptotic gene, B-cell lymphoma-2 (Bcl-2) is one of a complex group of genes located on chromosome 18 which control programmed cell death by preventing cells from responding to stimuli that initiate apoptosis and thereby may influence tumor prognosis. The bcl-2 is residing in the mitochondria and forms homo and hetero dimers with another protein in this family, Bax. Bcl2 is expressed in the normal brain tissues, gliosis and gliomas (Grade I, II, and III) but lack in grade IV [10].

\section{Cases:}

\section{MATERIAL AND METHODS}

This is a retrospective study; it was included 50 cases of CNS lesions that were retrieved from the neuropathology archives of National cancer institute (NCI) and from pathology department faculty of medicine, Zagazig University in the period from September 2009 to September 2012. Astrocytomas specimens were obtained by stereotactic biopsies; astrocytosis specimens were obtained by resections; they fixed in $10 \%$ formalin. The clinical data of the patients were obtained from the medical files.

\section{Histopathological study:}

Consecutive 4um sections were prepared and stained with hematoxyline \& eosin for histopathologic exami-nation. Pathological diagnosis was made according to the latest WHO grading system (2007) [11].

\section{Immunohistochemical Study:}

The immunohistochemical reac-tions were carried out using streptavidin- biotin immunoperxidase staining technique (Dako- Cytomation, Glostrup, Denmark) Paraffin sections were cut at $5 \mu$ thickness, deparaffinized by incubating them in the oven(Panathonic) at $56^{\circ} \mathrm{C}$ for 15 minutes, inserted in xylene for 30 minutes and rehydrated in descending grades of alcohol at room temperature. Thereafter, blocking of endogenous peroxidase activity with 0.3 $\%$ hydrogen peroxide in water for 30 minutes at room temperature was carried out. Antigen retrieval was performed using Dako target retrieval solution (PH 6.0) (EZ retrival system, Biogenic, Fremount, CA, and USA.); boiled in a microwave. Slides were incubated with primary antibodies for 30 minutes at room temperature; antibodies used were; anti IDH rabbit monoclonal antibody (clone H09, 1:50 dilution; Cell signaling Technology, Dinova, Hamburg, Germany). Anti EGFR mouse monoclonal antibody (clone 2-18C9, 1:50 dilution; Dako, Carpinteria, USA). Anti $\mathrm{Bcl} 2$ mouse monoclonal antibody (clone 100/D5, 1:50 dilution; Dako, Corp, Santa Barara, CA, USA). Incubation with secondary antibody were performed then product visuali-zation by incubated with diaminobenzidine (DAB) substrate as a chromagen (Dako-
Cytomation, Glostrup, Denmark); for 5-10 minutes. Sections were counterstained with Mayer's hematoxylin for (2-5) minutes (BioGenex Laboratories, San Ramon, CA), and washed once each with distalled water and PBS. Diffuse fibrillary astrocytoma, Colon carcinoma and Tonsil (reactive hyperplasia) were used as a positive control for IDH1, EGFR and $\mathrm{Bcl} 2$ respectively. Negative controls were obtained by omission of primary antibodies.

\section{Interpretation and evaluation of immunostaining:}

\section{1-Evaluation of IDH1immunostaining:}

Standard m IDH1staining is granular cytoplsmic in distribution with red/brown color. The degree of immunohistochemical reactivity was classified into one of the three semi quantitative categories: 1) Negative; none of glioma cells express mIDH1, 2) Focal positive; presence of more than five IDH1 positive glioma cells in less than $10 \%$ of tumor cells, 3) Diffuse positive; the area fraction of IDH1 positive glioma cells is more than $10 \%$ of tumor cells. All cases classified as focal and diffuse positive were considered as mutant IDH1 positive cases. Wild type of IDH1 did not capture the stain and considered as negative cases [12].

\section{2-Evaluation of EGFR immunostaining:}

EGFR immunostaining were evaluated according to the semi quantitative scoring approach for the intensity of membranous staining (weak, moderate or strong), the quality (partial or complete membranous staining) and for the proportion of stained cells. It includes score 0, 1, 2 and score 3 . Score 0 ; none of the tumor cells showed membranous staining (Cytoplasmic staining of the tumor cells was not regarded and considered as negative). Score 1; faint (intensity) and partial (quality) membrane staining in more than $10 \%$ of tumor cells. It was regarded as weak positive. Score 2; moderate and complete membrane staining in more than $10 \%$ of tumor cells. It was considered as moderate positive. Score 3; strong and complete membrane staining in more than $10 \%$ tumor cells. It was regarded as strong positive. Score of 0 and 1 were considered as negative and a score of 2 and 3 as positive cases [13].

\section{3-Evaluation of bcl 2 immunostaining:}

$\mathrm{Bcl} 2$ is positive as diffuse golden yellow cytoplasmic staining (sites of the mitochondria). The bcl-2 reactivity was calculated by counting 100 cells and expressed as percentage of positive cytoplasms from the total. Immunostaining was assessed by semi quantitative scoring system for percentage of cells staining and intensity of staining. Both were scored on scales of 0 to 3. : 1) Score 0; no stained cells were detected. 2) Score 1; mild staining in less than $25 \%$ of the cells. 3) Score 2; 
moderate staining in 25 to $50 \%$ positivity.4) Score 3; more than $50 \%$ of cells showed marked positivity [14].

Statistical analysis: the association of molecular markers were tested by Chi-square test and Fisher's exact test. The statistical significance was defined as $\mathrm{P}$-value $=0.5$. P-value $<0.05$ was considered significant, P-value $<0.001$ was considered highly significant and $\mathrm{P}$-value $>0.05$ was considered non signi-ficant.

\section{RESULTS:}

Fifty representative tissue blocks were studied retrospectively. The histopath-ological diagnosis were performed ; it was included diffuse fibrillary astrocytomas WHO grade II representing 23/50
(46\%), oligoastr-ocytoma WHO grade II $2 / 50$ (4\%) and gemistocytic astrocytoma WHO grade II 5 /50(10\%). Astrocytosis lesions were included brain abcesses 4/50 (8\%), gliosis around astrocytoma 4 $150(8 \%)$, gliosis adjacent to meningioma $3 / 50(6 \%)$, gliosis surround metastases $3 / 50(6 \%)$, aneurismal cyst with hemorrhage $2 / 50(4 \%)$, piloid reactive gliosis $1 / 50(2 \%)$ and angioma with cerebral hemorrhage $3 / 50(6 \%)$. The age of studied cases was ranged from 5 to 72 with the mean 33.6 \pm 22.7 . Regarding to sex distribution among the studied cases, the majority of cases were male (72\%); astrocytomas were (44\%) and gliosis lesions were $(28 \%)$.

Table (1): Histopathologic diagnosis of the studied cases.

\begin{tabular}{lcc}
\hline \multicolumn{1}{c}{ DIAGNOSIS } & NO. & \% \\
\hline Astrocytomas Cases : & $\mathbf{3 0}$ & $\mathbf{6 0}$ \\
- Fibrillary astrocytoma & 23 & 46 \\
- Gemistocytic strocytoma & 5 & 10 \\
- Mixed oligoastrocytoma & 2 & 4 \\
Gliosis lesions: & $\mathbf{2 0}$ & $\mathbf{4 0}$ \\
- Brain abcesses & 4 & 8 \\
- Margin of astrocytoma & 4 & 8 \\
- Margin of meningiomas & 3 & 6 \\
- Anrgin of metastases & 3 & 6 \\
- Pilourismal reactive gliosis & 2 & 4 \\
- Angioma and cerebral hemorrhage & 1 & 2 \\
Total & 3 & 6 \\
\hline
\end{tabular}

The majority of studied cases were diffuse fibrillary astrocytomas (46\%)

Table (2) IDH1 expression in studied cases:

Mutant IDH1 protein was expressed in $18(60 \%)$ out of 30 WHO grade II astrocytomas, but entirely absent in all 20 reactive astrocytosis samples.

\begin{tabular}{lcccccccccc}
\hline & \multicolumn{4}{c}{ Positive interpretation } & \multirow{2}{*}{$\begin{array}{c}\text { Negative } \\
\text { interpretation }\end{array}$} & $\begin{array}{c}\text { Fisher's } \\
\text { exact test } \\
\text { P value }\end{array}$ \\
\cline { 2 - 9 } & No & Focal & Diffuse & Total & No & \% & \\
\cline { 2 - 9 } & & No & $\%$ & No & $\%$ & No & $\%$ & No & \\
\hline Astrocytomas cases & 30 & 5 & 16.7 & 13 & 43.3 & 18 & 60 & 12 & 40 & $0.00014^{* *}$ \\
Astrocytosis lesions & 20 & 0.0 & 0.0 & 0.0 & 0.0 & 0 & 0 & 20 & 100 & \\
\hline
\end{tabular}

Negative: no stained cells were detected. Positive: presence of stained cells,

$* *$ Highly significant $(\mathrm{P}-$ value $<0.001)$.

Positive Bcl 2 expressions were detected in 17 cases $(56.7 \%)$ of examined astrocytomas and positive in 15 cases $(75 \%)$ of astrocytosis lesions. No relation was found between $\mathrm{Bcl} 2$ expression in astrocytomas and astrocytosis 
Table (3) Bcl2 expression in all studied cases.

\begin{tabular}{lcccc}
\hline & No & $\begin{array}{c}\text { Negative } \\
\text { interpretation }\end{array}$ & $\begin{array}{c}\text { Positive } \\
\text { interpretation }\end{array}$ & $\begin{array}{c}\text { Fisher's exact } \\
\text { test P-value }\end{array}$ \\
\hline Astrocytoma cases & 30 & $13(43.3 \%)$ & $17(56.7 \%)$ & 0.18 \\
astrocytosis lesions & 20 & $5(25 \%)$ & $15(75 \%)$ & N.S \\
Total & 50 & $18(36 \%)$ & $32(64 \%)$ & \\
\hline
\end{tabular}

N.S: Non significant.

Negative: no stained cells.

Positive: presence of stained cells.

EGFR protein expression was detected in 16 cases (53.3\%) out of 30 astrocytomas cases; fifteen of them (50\%) were moderate and strong positive (score 2 and 3) but only one case showed weak positivity (score 1). EGFR expressions in astrocytosis were detected in only 5 cases $(25 \%)$ that were weak positive.

Table (4) EGFR expression in all studied lesions

\begin{tabular}{lcccc}
\hline & No & $\begin{array}{c}\text { Negative } \\
\text { interpretation } \\
\text { (score 0 and 1) }\end{array}$ & $\begin{array}{c}\text { Positive } \\
\text { interpretation } \\
\text { (score 2 and 3) } \\
15(50 \%)\end{array}$ & $\begin{array}{c}\text { Fisher's } \\
\text { exact test } \\
\text { P- value }\end{array}$ \\
$\begin{array}{l}\text { Astrocytomas cases } \\
\text { astrocytosis lesions }\end{array}$ & 30 & $15(50 \%)$ & 0.0 & $0.0000149 * *$ \\
Total & 20 & $20(100 \%)$ & $15(30 \%)$ & \\
\hline
\end{tabular}

Negative include score 0 and score 1.

Positive include score 2 and score 3. ** Highly significant $(<0.001)$.

Table (5) Summary of immunohistochemical expression for IDH1, BCL2 and EGFR in all studied lesions

\begin{tabular}{lcccccc}
\hline & \multicolumn{2}{c}{ IDH1 } & \multicolumn{2}{c}{ BCL2 } & \multicolumn{2}{c}{ EGFR } \\
\cline { 2 - 7 } & $\begin{array}{l}\text { Positive } \\
\text { interpretation }\end{array}$ & $\begin{array}{l}\text { Negative } \\
\text { interpretation }\end{array}$ & $\begin{array}{l}\text { Positive } \\
\text { interpretation }\end{array}$ & $\begin{array}{l}\text { Negative } \\
\text { interpretation }\end{array}$ & $\begin{array}{l}\text { Positive } \\
\text { interpretation }\end{array}$ & $\begin{array}{l}\text { Negative } \\
\text { interpretation }\end{array}$ \\
\cline { 2 - 8 } $\begin{array}{l}\text { Astrocytomas } \\
\text { cases }\end{array}$ & $12(40 \%)$ & $18(60 \%)$ & $13(43.3 \%)$ & $17(56.6 \%)$ & $15(50 \%)$ & $15(50 \%)$ \\
$\begin{array}{l}\text { Astrocytosis } \\
\text { lesions }\end{array}$ & $20(100 \%)$ & 0.0 & $5(25 \%)$ & $15(75 \%)$ & $20(100 \%)$ & 0.0 \\
Total & $32(64 \%)$ & $18(36 \%)$ & $18(36 \%)$ & $32(64 \%)$ & $35(70 \%)$ & $15(30 \%)$
\end{tabular}

Fisher's

exact test p- $\quad=0.00014 * * \quad 0.18$ N.S $\quad=0.000149 * *$

value

** Highly significant $<0.001$. N.S: Non significant

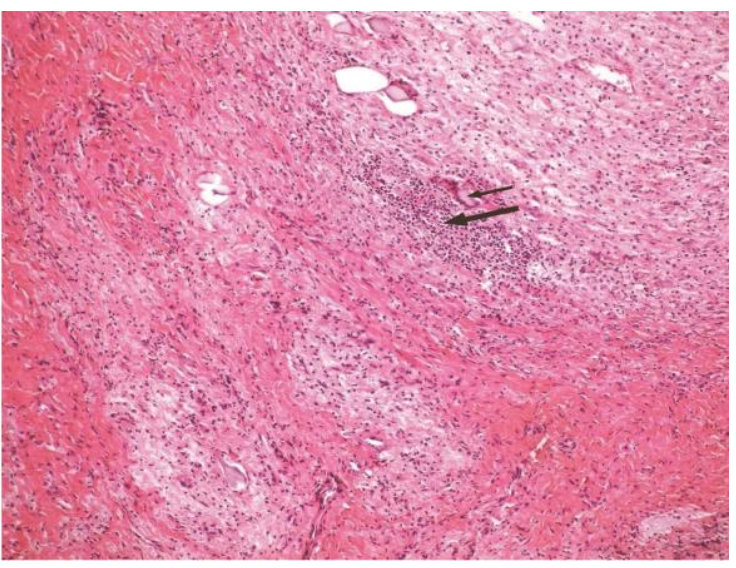

Fig. (1a): Brain abcess surrounded by cellular reactive astrocytosis; with inflammatory cells (thick arrow) and giant cells (thin arrow) (H\&E, X 100).

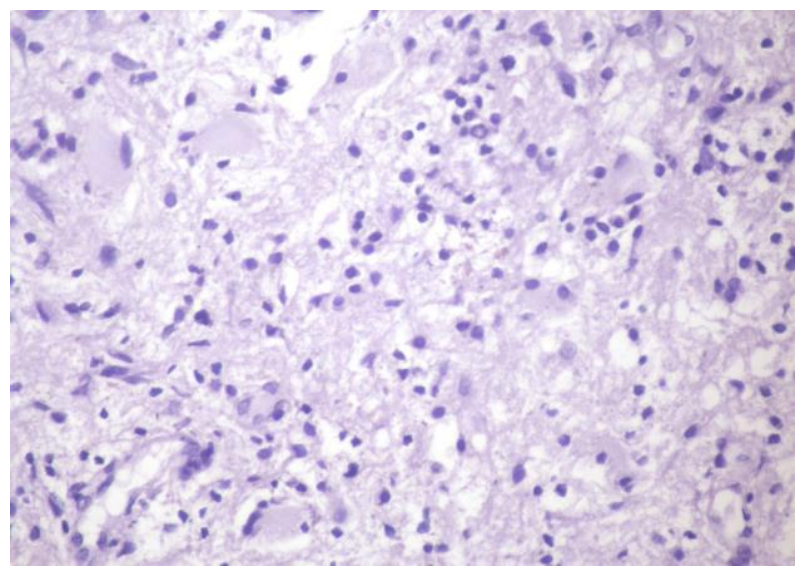

Fig. (1b): Immunohistochemical staining for IDH1 of brain abcess surrounded by cellular reactive astrocytosis: show negative immunoreactivity(Immunoperoxidase staining, DAB chromogen, Mayers hemato-xyline counter stain X400). 


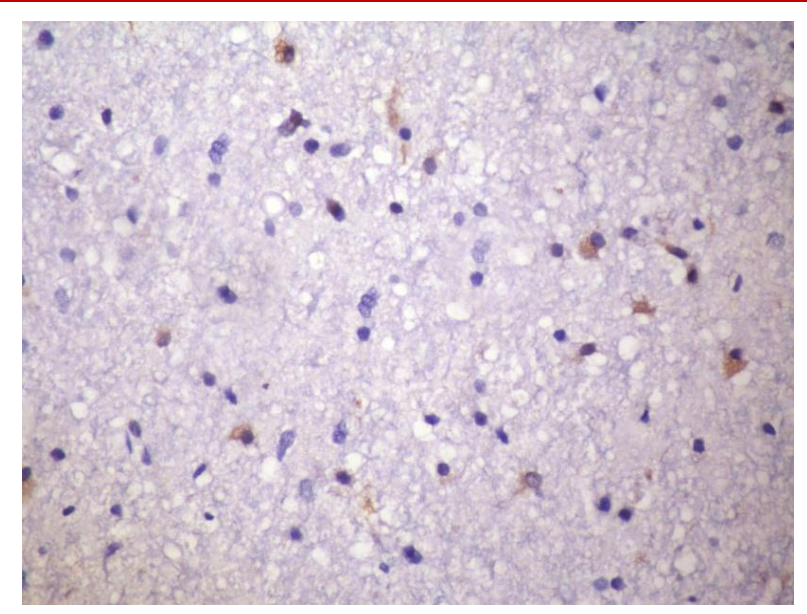

Fig. (2a): Immunohistochemical staining for $\mathrm{Bcl} 2$ of cellular reactive astrocytosis show; mild cytoplasmic immunoreactivity in less than $10 \%$ (score 1). (Immunoperoxidase staining, DAB chromogen. Mayers hematoxylin Counter stain X 400).

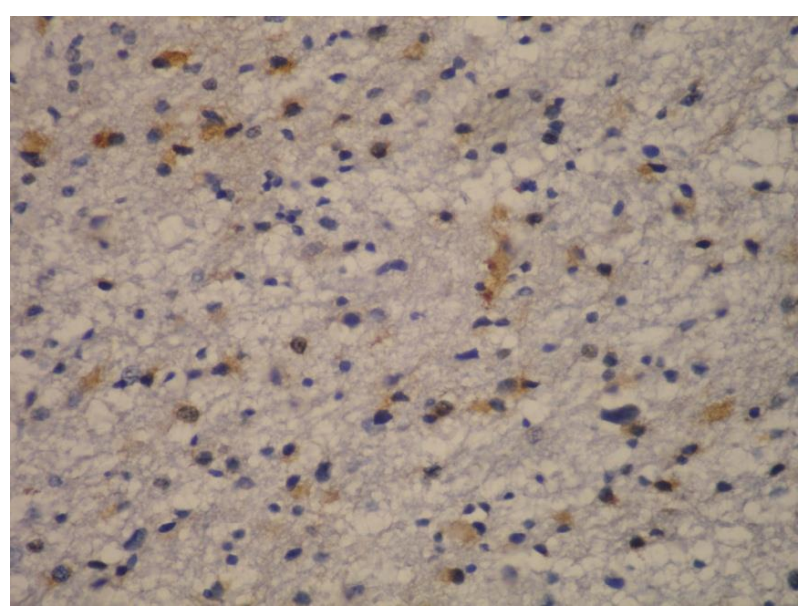

Fig. (2b): Immunohistochemical staining for $\mathrm{Bcl} 2$ in reactive astrocytosis show; moderate bcl2 cytoplasmic staining in about $30 \%$ (score2). (Immunoperoxidase staining, DAB chromogen. Mayers hematoxylin Counter stain $\mathrm{x}$ 400).

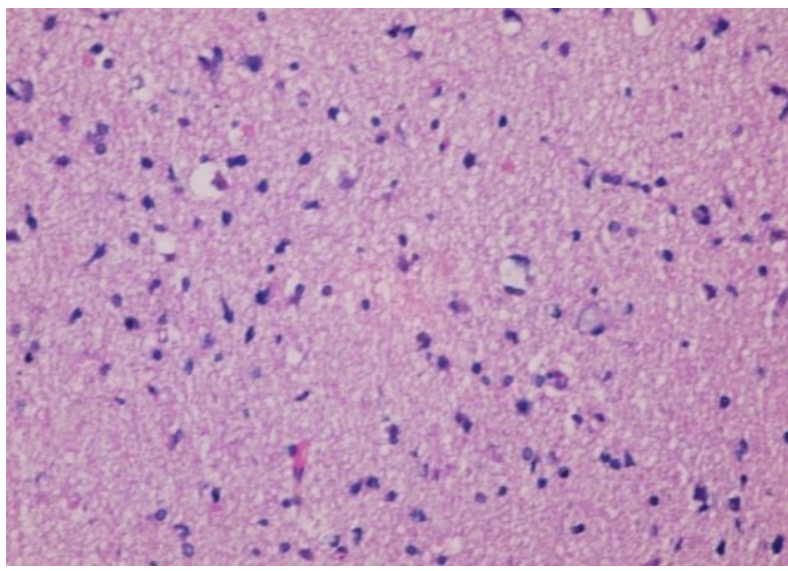

Fig. (3): Diffuse fibrillary astrocytoma WHO grade II showing moderate cellularity with occasional mild pleomorophic hyperchromatic irregular nuclei and glial fibrillary background (H\&E, X400)

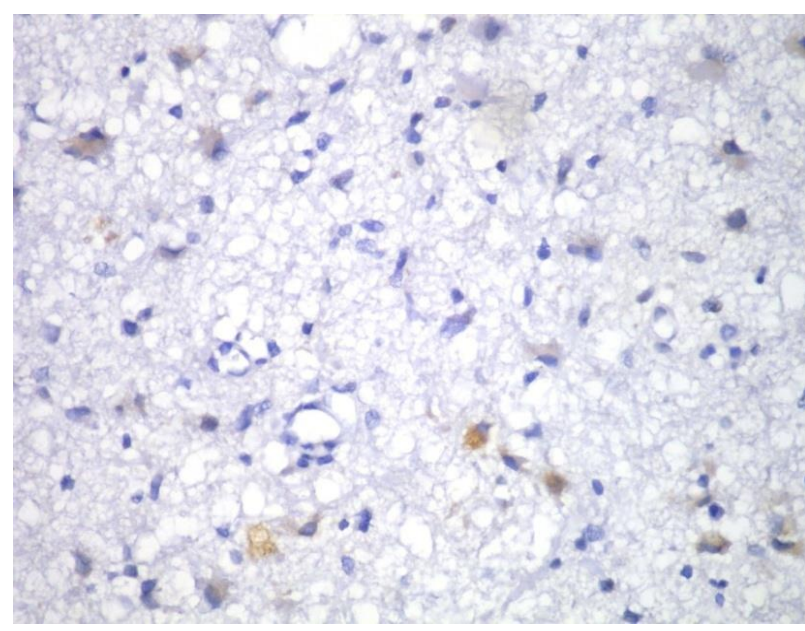

Fig. (4a): Immunohistochemical staining of IDH1 in a case of diffuse fibrillary astrocytoma WHO grade II show; focal granular cytoplasmic immunoreactivity in less than $10 \%$ of cells (Immunoperoxidase staining, DAB chromogen. Mayers hematoxyline counter stain x 400).

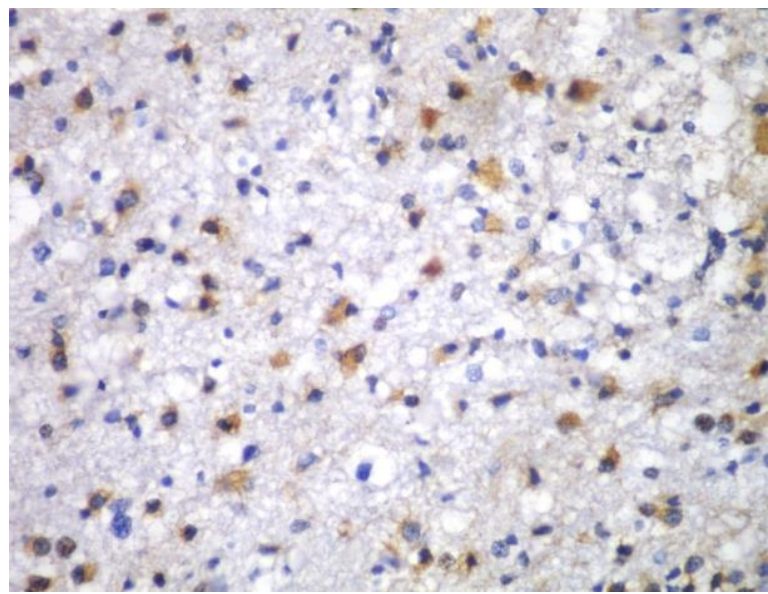

Fig. (4b): Immunohistochemical staining of IDH1 in diffuse fibrillary astrocytoma WHO grade II showing; diffuse granular cytoplasmic immunoreactivity in more than $10 \%$ of cells (Immune peroxidase staining, DAB chromoge, Mayers hematoxyline counter stain x 400). 


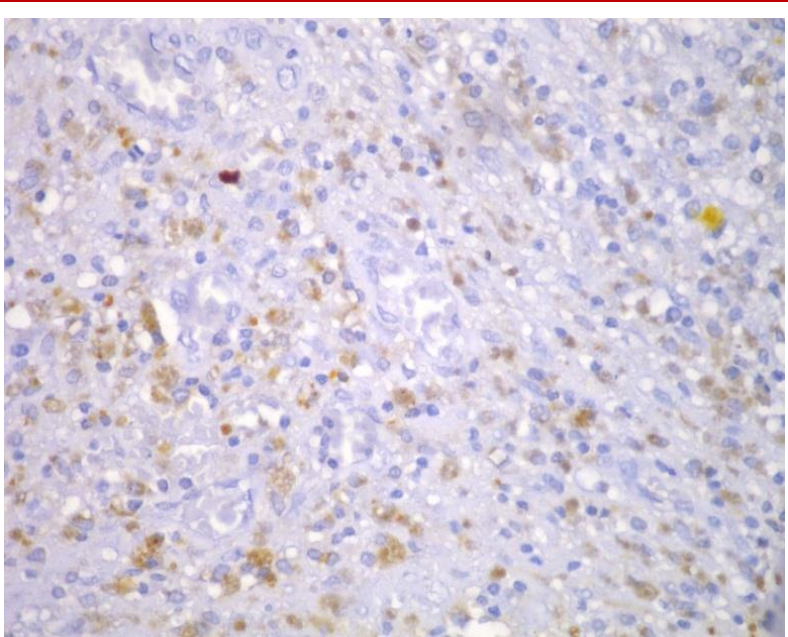

Fig. (5a): Immunohistochemical staining of $\mathrm{Bcl} 2$ in a case of diffuse fibrillary astrocytoma WHO grade II show; moderate cytoplasmic immunoreactivity; score 2 (25-50\% positive reaction) (immunoperoxidase staining, DAB chromogen. Mayers hematoxyline counter stain $\mathrm{x}$ 400).

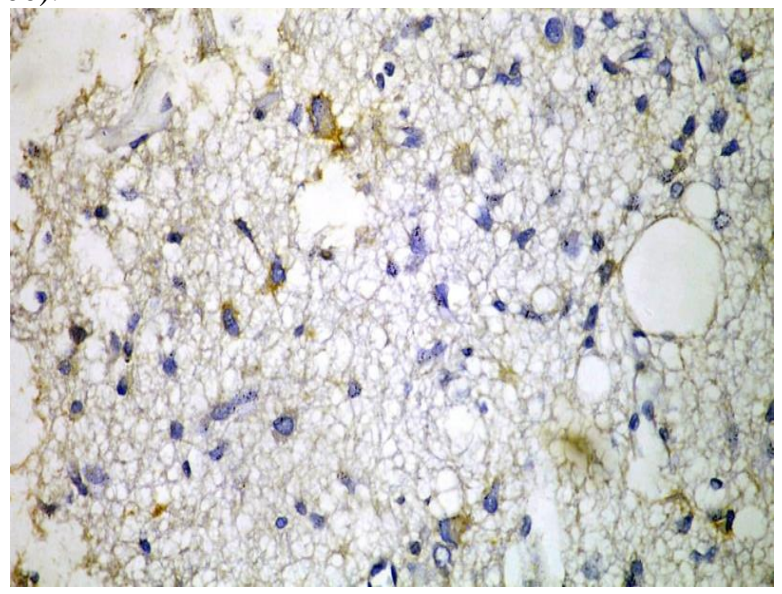

Fig. (6a): Immunohistochemical staining of EGFR of diffuse fibrillary astrocytoma WHO grade II show; moderate and complete membranous immunoreactivity, score2 (Immunoperoxidase staining, DAB chromogen. Mayers hematoxyline counter stain x 400).

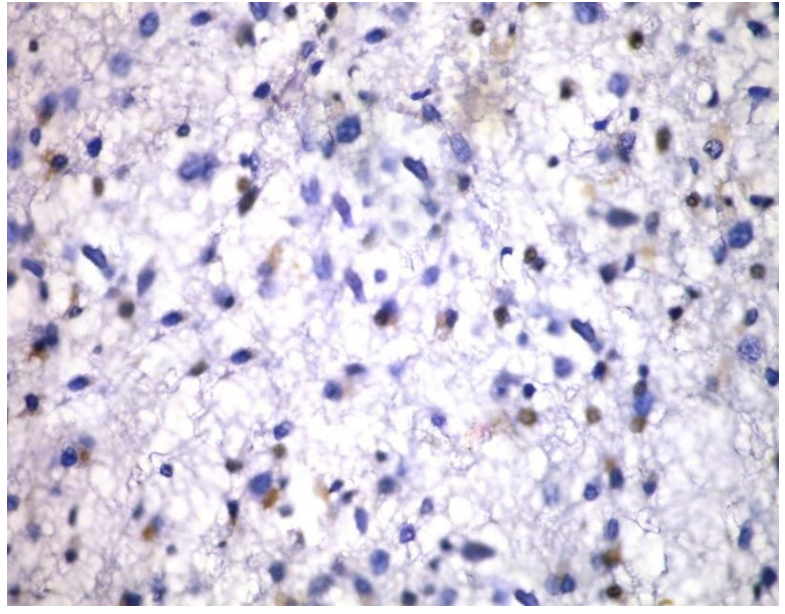

Fig. (5b): Immunohistochemical staining of $\mathrm{Bcl} 2$ in a case of diffuse fibrillary astrocytoma WHO grade II show mild cytoplasmic immunoreactivity; score $1 \quad<25 \%$ positive reaction) (Immunoperoxidase staining, DAB chromogen. Mayers hematoxyline counter stain x 400)

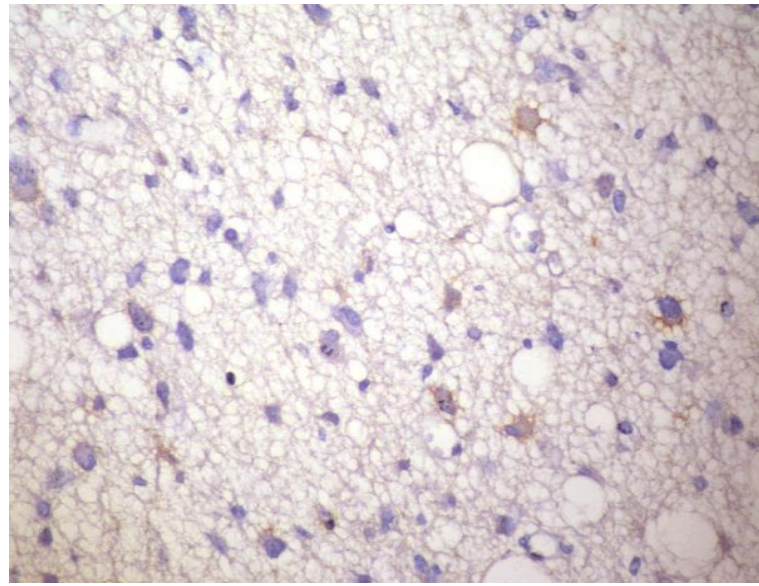

Fig. (6b): Immunohistochemical staining of EGFR of diffuse fibrillary astrocytoma WHO grade II show; faint and partial membranous immunoreactivity, score1 (faint and partial membranous $<10 \%$ tumor cells) (immuneperoxidase staining, DAB chromogen. Mayers hematoxyline Counter stain x 400).

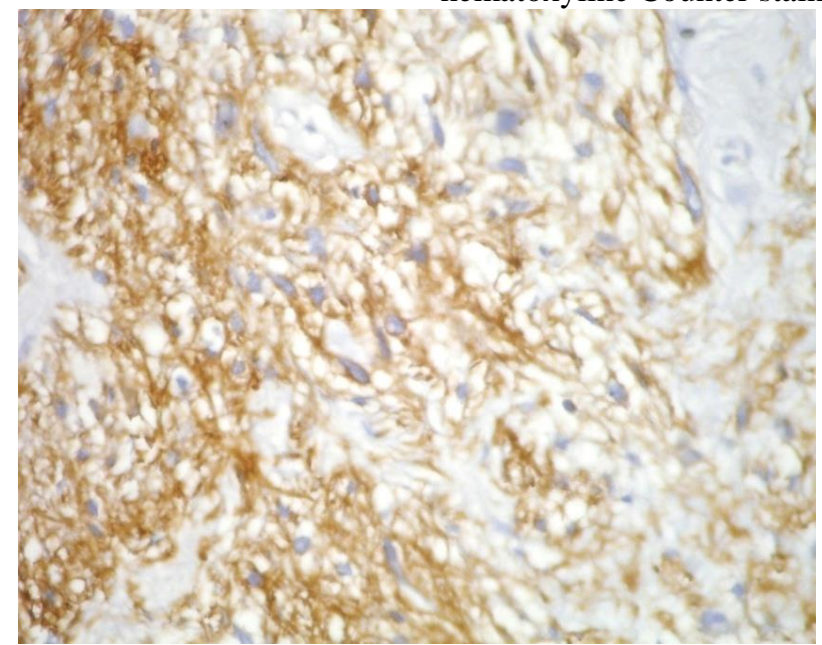

Fig. (6c): Immunohistochemical staining of EGFR of diffuse fibrillary astrocytoma WHO grade II show; strong and complete membranous immunoreactivity, score 3 ( strong and complete membranous in more than $10 \%$ of tumor cells) (Immunoperoxidase staining, DAB chromogen. Mayers hematoxyline counter stain x 400). 


\section{DISCUSSION}

Gliomas are the most relevant primary brain tumors; representing about $81.7 \%$ of cases. Diffuse astrocytomas are the most common type of gliomas representing about $67.6 \%$ of gliomas and $5-10 \%$ of astrocytic tumors (Ibrahim et al., 2011)[2]. The distinction of diffuse astrocytomas from astrocytosis is one of the most difficult differential diagnosis faced by surgical pathologists especially with small biopsies (e.g. sterotactic biopsy). Sometimes it is difficult to differentiate reactive from neoplastic astrocytes. This justifies the need to specific markers for neoplastic glial cells. Each of the commonly used approaches to address this differential diagnosis such as ki-67 and TP53 has problems with sensitivity and specificity (Habberstad et al., 2011).[15]

IDH1 is a genetic hallmark of grade II diffuse astrocytoma; it occurs as an early step of astrocytic tumor genesis (Sipayya et al., 2012)[16]. The use of an antibody specific to mutant IDH1 (m IDH1) is of both diagnostic and prognostic relevance in astrocytomas. Immunohistochemical reactivity is more sensitive than other detection methods such as DNA extraction and sequencing to detect tumors with such mutation especially in the very small biopsies (Preusser et al., 2011).[12]

In the present study, the age of the studied cases ranged from 5 to 72 with a mean of 33.6 \pm 22.7 . The mean of pediatric group was 11 years, and that of adult group was 50 years.

The current study revealed that $\mathrm{m}$ IDH1 has high sensitivity and specificity to distinguish low grade diffuse astrocytoma from astrocytosis $(\mathrm{P}$ - value $=0.000149)$. IDH1 was totally absent in astrocytosis (100\%) and was expressed in $60 \%$ (18/30) of studied diffuse astrocytomas. The staining of all cells permits the identification of single infiltrating cells in otherwise brain tissue. All stained cells were considered as neoplastic cells that have mutant IDH1 while reactive astrocytes with wild type IDH1 did not capture any staining.

The results of the present study were close to the results of previous studies by Camelo pirague et al., 2011[17], Sipayya et al., 2012[16], Kuriana et al.,2013[18] and Lewandowska et al., 2014[19] who revealed that m IDH1 was highly sensitive in the differential diagnosis of low grade diffuse astrocytomas WHO grade II from astrocytosis where it was absolutely absent in reactive conditions and present in (47.6\% [10/21],42.5\%[19/45] , 54\%[25/46] and
$56 \%[42 / 75]$ ) of diffuse astrocytomas WHO grade II cases.

Nearly similar results were reported by previous studies of Blass et al., 2008[20], Capper et al., 2009[21], Hartmann et al., 2009[22] ,Chang et al., 2011[23] and Mellai et al.,2012[24] where they found $68 \%, 70 \%, 72.7 \%$, $78.3 \%$ and $75 \%$ of studied diffuse astrocyomas cases WHO grade II expressed m IDH1. This discrepancy to the current results could be explained by different specimen preparation procedures and different sample size where Blass et al., 2008 studied 685 brain tumors with 93 diffuse astrocytomas cases and Hartmann et al., 2009 studied 1010 of diffuse astrocytomas. Mellai et al., 2012 performed their study on 608 cases and found that 455 of them expressed IDH1.

The current results were different from Capper et al., 2010[25] who found that $83 \%$ of their studied 345 diffuse astrocytomas cases were positive expressed $\mathrm{m}$ IDH1. This discrepancy with the current results may be attributed to the different used antibody clone. In the present study anti IDH1 rabbit monoclonal antibody; clone H09 was used; while Capper et al., 2010 used mIDH1R132H clone H14. Also Capper et al., 2010 considered the immunoreactivity positive when tumor cells showed strong cytoplasmic staining, even if only one positive cell was detected. However, in this study, cases with more than five positive cells is necessary to be considered as positive cases either focal; if involve less than $10 \%$ of tumor cells or diffuse; if involve more than $10 \%$ of tumor cells. But regarded to astrocytosis; it was in agreement to the present study that reactive astrocytes did not capture any staining.

The results of the present study were also different from previous study performed by Agarwal et al., 2013[26] who assessed IDH1 mutation in $88 \%$ of diffuse astrocytoma cases. So they suggested that IDH1 occurs as an early event in glioma genesis. These differences may be attributed to different number of included cases where they studied fifty cases while in the present study; astrocytomas were thirty cases.

A controversial result was given by Takano et al., 2011[27] who reported that; IDH1 expression in diffuse astrocytoma grade II were $100 \%$; these results were different from the current results that may be due to different used antibody clone; where they used clone MT 3.1 (Neomarker, Westinghouse, Fremont, CA, USA); while in the present study anti IDH1 rabbit monoclonal antibody clone H09 (Cell signaling Technology, Dinova, Hamburg, Germany) was 
used. Also their study involved only four cases; this may be other cause. Other different result was reported by Thota et al., 2012 [28] who found also that $100 \%$ of their studied diffuse astrocytomas (12/12) were positive for $\mathrm{m}$ IDH1.

As regarded to oligoastro-cytoma WHO grade II cases, $\mathrm{m}$ IDH1 was diffusely expressed in the studied two cases $(100 \%)$. This is in agreement with Yan et al., 2009 [29] who found all studied oligoastrocytoma cases 10/10 (100\%) stained diffusely with IDH1. These results were also in agreement to Bourne and Schiff 2010 [30] who reported that IDH1 expression in mixed oligoastrocytomas was ranged from $50 \%$ to $100 \%$.

These results of the present study were close to results of other studies by Blass et al.,2008[20] , Capper et al., 2009[21], Hartmann et al .,2009[22], Watanable et al., 2009[31] and Mellai et al.,2012[24] whereas they clarify that $78 \%, 90 \%, 81.6 \%, 94 \%$ and $72 \%$ of their studied oligoastrocytic tumors WHO grade II stained with IDH1.

Concerning to the importance of IDH1 as a prognostic factor; Hartmann et al.,2010[32] arranged the order of outcome of astrocytic tumors according to the relation of IDH1mutation and overall survival from the most favorable to least favorable one as :DFA WHO grade II with IDH1 mutation, DFA WHO grade II without IDH1 mutation, anaplastic astrocytoma WHO grade III with IDH1 mutation, GBM WHO grade IV with IDH1 mutation then anaplastic astrocytoma WHO grade III without IDH1 mutation, GBM WHO grade IV without IDH1 mutation.

The prognostic value of IDH1 is also showed in differentiating oligoastrocytoma with predominant astrocytic component from oligodendroglioma with surround reactive gliosis which has a better prognosis. IDH1 assists in separating oligodendroglial tumors from neurocytomas and other clear cell tumors (Sipayya et al., 2012)[16].

As regard to epidermal growth factor receptor expression (EGFR); it was highly significant in distinguishing diffuse astrocytoma WHO grade II from reactive astrocytosis (P value $=0.00014)$. It was entirely absent in reactive astrocytosis lesions (100\%); weakly expressed in five cases that were considered as negative cases. It was expressed in 50\% (15/30) of low grade diffuse astrocytomas. One case was weakly expressed; that was omitted.

Vandenbos et al., 2011[33] reported that EGFR expression is a useful diagnostic tool in the differential diagnosis of low grade diffuse astrocytoma and reactive astrocytosis. EGFR expressed moderate and strong staining in 100\% of their studied diffuse astrocytoma cases and weakly expressed in $82 \%$ of reactive astrocytosis. These discrepancies in the results may be attributed to the difference in the nature of studied gliosis lesions where in the current study; the previous history of gliosis lesions before surgery was unknown. EGFR expression is variable among acute, subacute and chronic cases. Also this may be explained by different antibodies used whereas several antibodies for EGFR are available but their specificity can differ. Some antibodies recognize only wild type EGFR; others are specific for mutated form EGFRVIII and some antibodies recognize both forms as used in the current study.

These results were close to $\mathrm{Hu}$ et al., 2013[34] who reported that $40.6 \%$ of their studied diffuse astrocytoma WHO II expressed EGFR either moderate (score 2) and marked EGFR staining (score 3) while weak positivity (score 1) was omitted. This difference with the results of the present study may be due to different used scoring systems where Hu et al., 2013 considered the score 2 when $25-50 \%$ positive cells were detected and score 3 when more than $50 \%$ positive cells present. But in the present study; score 2 was considered as moderate and complete membrane staining in more than $10 \%$ of tumor cells; score 3 as strong and complete membrane staining in more than $10 \%$ tumor cells.

Nearly similar results were reported by Marquez et al., 2004[35] who found that EGFR protein level was $42 \%$ of diffuse astrocytoma cases. This discrepancy of the results with the present work may be attributed to the use of different antibodies, specimen preparation procedures, criteria defining positive cases and methodological heterogeneity of the determination of this receptor protein.

Larysz et al., 2011[36] reported that $33.3 \%$ of their studied diffuse astrocytomas cases were positive for EGFR. This discrepancy may be due to different used antibody whereas the immunoreactivity against either the external or the internal domain of the receptor is different; the external domain is more vulnerable to fixation and tissue processing than the internal one. Antibodies directed against the external domain recorded higher expression that is used in the present study. Different sample size may play a role; whereas they performed their study on seven cases only of diffuse astrocytoma WHO II while in the present study; studied cases were thirty. 
As regard to the studied two cases of oligoastrocytoma. One of them was positive $(50 \%)$ as score 2 and the other was negative. But Puputti et al., 2006 [37] reported that EGFR expressed as 1 out of eight (11\%) of their studied oligoastrocytomas cases.

Avnider et al., 2006[38] reported that no relation was found between gemistocytic astrocytoma and EGFR protein expression but in the present study; one of the five cases of gemistocytic astrocytomas was positive for EGFR.

As regard to the $\mathrm{Bcl} 2$ expression in this work; there was no significant value between bcl2 expression among astrocytomas cases and astrocytosis lesions. Bcl2 was expressed in 56.7 $\%(17 / 30)$ of diffuse astrocytomas cases and in $75 \%(15 / 20)$ of astrocytosis. This is in agreement with Alderson et al., 1995[39] and RodriguezPereira et al., 2001 [40] who reported that 57\% of their studied diffuse astrocytomas cases were positive for bcl2. Also Newcomb et al., 1997[41] found $57 \%$ (12/21) of pediatric and 73\% (27 / 37 ) of the adult cases positive for bcl 2 .

The result of this study goes with the results of previous studies by (Rieger $\boldsymbol{e t}$ al. 1998[42]; Martin et al. 2001[43]; RodriguezPereira et al. 2001[40] ) who reported that Bcl2 expressed in astrocytosis as well as diffuse astrocytomas. These findings suggest that: (i) the glial cells may have a prolonged survival; (ii) apoptosis occurs in gliotic tissues indicating that BCL-2 plays a role in its formation.

These results were close to studies performed by Hussien et al., 2006[44] who found $54.2 \%$ of grade II diffuse astrocytoma and $75 \%$ of gliosis cases positive for bcl2.

Dietzmann et al. 1996 [45] and Fels et al. 2000[46] reported that Bcl2 was expressed in only $25.4 \%$ of diffuse astrocytoma WHO II. This discrepancy might be due to the genetic heterogeneity of gliomas.

\section{REFERENCES}

1. McKean-Cowdin R., Razavi P. and Preston-Martin S.( 2008): Brain Tumours. International Ency-clopedia of Public Health ; 338-47.

2. Ibrahim A.S., Mikhail N.N., Darwesh H. and Heikel T. (2011): Egypt National Cancer Registry, Damietta Profile - 2009.

3. Inskip P. D. and Rone (2001): Brain and Other Central Nervous System Cancer .111-2.

4. Camelo-Piragua S., Jansen, M., Ganguly A., Kim J.C., Cosper A.K., Dias-Santagata D., Nutt C.L., A. John Iafrate and Louis D.N. (2011): A Sensitive and Specific Diagnostic Panel to Distinguish Diffuse Astrocytoma from Astrocytosis: Chromosome 7 Gain with Mutant Isocitrate Dehy-drogenase 1 and p53. J Neuropathol Exp Neurol; 70 (2): 110-5.
5. Lawrence F. E., Ghirnikar R. S. and Lee Y. L. (2000): Glial Fibrillary Acidic Protein: GFAP-Thirty-OneYears (1969-2000). Ne-urochemical Research; 25: 1439-51.

6. Mahzouni P., Mokhtari M. and Amirmansour B. (2007): Different-iation between reactive gliosis and astrocytomas by MIB-1/ki67 imm-unostaining. Accepted: 26.8.

7. Lammie G.A., Beckett A., Courtney R. et al., (1994) : An immunohistochemical study of p53 and proliferating cell nuclear antigen expression in progressive multifocal leukoencephalopathy. Acta Neuro-patholo; 88:564-7.

8. Yan H., Parson D. W., Jin G. et al., (2009): IDH1 and IDH2 in glioma. N Engl J Med; 360:765-73.

9. Larysz D., Kula D., Kowal M., RudnikA., JarząbM. et al., (2011): Epidermal growth factor receptor gene expression in high grade Gliomas. Folia Neuropathol; 49 (1): 28-38.

10. McDonald F. E., Ironside J. W., Grego A. et al., (2002): The prognostic influence of bcl-2 in malignant glioma. British Journal of Cancer. 86, 1899 - 904.

11. Scheithauer B. W., Fuller G. N. and Vanden Berg S. R. (2008): The 2007 WHO Classification of Tumors of the Nervous System: Contro-versies in Surgical Neuropathology. Brain Pathology; 18: 307-16.

12. Preusser A., Wohrer S., Stary R., Hoftberger B., Streubel J. A. and Hainfellner (2011): Value and limitations of immunohistoch-emistry and gene sequencing for detection of the IDH1-R132H mutation in diffuse glioma biopsy specimens,. J. Neuropathol. Exp. Neurol ; $70: 715-23$.

13. Ambroise M. M., Khosla C., Ghosh M., M allikarjuna V.S. and Annapurneswari S. (2010): The role of immunohistochemistry in predicting behavior of astrocytic tumors. Asian Pac J Cancer Prev ; 11:107984.

14. McDonald F. E., Ironside J. W., Grego A. et al., (2002): The prognostic influence of bcl-2 in malignant glioma. British Journal of Cancer. 86, $1899-904$.

15. Habberstad A.H., Gulati S. and Torp S.H. (2011) : Evaluation of the proliferation markers Ki-67/MIB-1, mitosin, survivin, $\mathrm{pHH} 3$, and DNA topoisomerase II $\mathrm{a}$ in human anaplastic astrocytomas - an immunohistochemical study. Diagnostic Pathology; $6: 43$

16. Sipayya V., Sharma I., Sharma K. C. and Singh A. (2012): Immuno-histochemical expression of IDH1 in gliomas: a tissue micro-array-based approach. J Cancer Res Ther ;8 (4): 598-601.

17. Camelo-Piragua S., Jansen M., Ganguly A., Kim J.C., Louis D.N., and Nut C.L. (2010): Mutant IDH1specific immunohistochemi-stry distinguishes diffuse astrocyt-oma from astroc-ytosis. Acta Neuro-pathol; 119(4): 509-511.

18. Kuriana M.K., Haynesa H.R., Crosbyb C., Hopkinsc K. and Williams M. (2013): Isocitrate dehydrogenase mutation analysis in gliomas as a diagnostic and prognostic biomarker ; 381:S61.

19. Lewandowska M.A., Furtak J., Szylberg T., Roszkowski K., Windorbska W., Rytlewska J. and Jozwick W. (2014): An Analysis of the Prognostic Value of IDH1 (Isocitrate Dehydrogenase 1) Mutation in Polish Glioma Patients. Mol Diagn Ther 18:45-53

20. Blass J., Meyer J., Mueller W., Korshunov A., Hartmann C., Von Deimling A (2008): Analysis of 
IDH1 codon 132 mutation in brain tumors. Acta Neuropathol ; 116:597-602.

21. Capper D., Zentgraf H., Balss J., von Deimling A. and Hartmann C. (2009): Monoclonal antibody specific for IDH1 R132H mutation . Acta Neuropathol; 118:599601.

22. Hartmann C., Meyer J., Balss J., Capper D., Mueller W., Christians A., et al. (2009): Type and frequency of IDH1 and IDH2 mutations are related to astrocytic and oligodendroglial differentiation and age: a study of 1,010 diffuse gliomas. Acta Neuropathol; 118: 469-74.

23. Chang C. M., Xu K. and Shu H. K. (2011) : The Role of Isocitrate Dehydrogenase Mutations in Glioma Brain Tumors, Molecular Targets of CNS Tumors . ISBN: 978-953-307-736-9. InTech, Available from: http:// www. intechopen. com/ books /molecular-targets-ofcns-tumors/ the-role-ofisocitrate-dehy-drogenasemutations-in-glioma-brain-tumors.

24. Mellai M., Caldera V., Annovazzi L. and Schiffer D.(2012): The Distribution and Significance of IDH Mutations in Gliomas.Chapter10; 299-442.

25. Capper D., Weibert S., Balss J., Habel A., et al., (2010) : Character-ization of R132H Mutation-specific IDH1 Antibody Binding in Brain Tumors. Brain Pathology; 20: 245-54.

26. Agarwal S, Sharma M.C., Jha P., Pathak P., Suri V. et al., (2013): Comparative study of IDH1 mutations in gliomas by immuno-histochemistry and DNA sequencing. Neuro Oncol; 10:1093.

27. Takano S., Tian W., Matsuda M., Yamamoto T., Ishikawa E., Kaneko M. K. and Yamazak K. (2011): Detection of IDH1 mutation in human gliomas: comparison of immunohistochemistry and sequencing. Brain Tumor Pathol; 10:1007-14.

28. Thota B., Shukla S.K., Srividya M.R. et al (2012): DH1 Mutations in Diffusely Infiltrating Astrocytomas Grade Specificity, Association With Protein Expression, and Clinical Relevance. Am J Clin Pathol. 138:177-84

29. Yan H., Parson D. W., Jin G. et al., (2009): IDH1 and IDH2 in glioma. N Engl J Med; 360:765-73.

30. Bourne T.D. and Schiff D. (2010): Update on molecular findings, management and outcome in low-grade gliomas. Nat Rev Neurol ;6 (12): 695-701.

31. Watanabe T., Nobusawa S., Kleihues P., and Ohgaki H. (2009) : IDH1 Mutations Are Early Events in the Development of Astrocytomas and Oligodendrogliomas . Am J Pathol. April; 174(4): 1149-53.

32. Hartmann C., Hentschel B., Wick W., Capper D., Felsberg J., Simon M., Westphal M., Schackert G., Meyermann R., Pietsch T. et al., (2010): Patients with IDH1 wild type anaplastic astrocytomas exhibit worse prognosis than IDH1 mutated glioblastomas and IDH1 mutation status accounts for the unfavorable prognostic effect of higher age: implications for classification of gliomas . Acta Neuropathol; 120:707-18.

33. Vandenbos F.B., Benchetrit M., Miquel C., Fontaine D. et al (2011): EGFR immunolabeling pattern may discriminate low-grade gliomas from gliosis. Neuro onco-logy J; 102:171-8.

34. Hu X., Weimiao, Zou Y, Zhang W., Zhang Y., and Hongyiliu ( 2013): Expression of p53, epidermal growth factor receptor, Ki-67 and O6-methylguanine-
DNA methyltransferase in human gliomas. Oncol Lett ; 6(1): $130-4$.

35. Marquez A., Wu R., Zhao J., Tao J. and Shi Z. (2004): Evaluation of epidermal growth factor receptor (EGFR) by chromogenic in situ hybridization (CISH) and immuno-histochemistry (IHC) in archival gliomas using bright-field micro-scopy. Diagn Mol Pathol ;13(1):1-8.

36. Larysz D., Kula D., Kowal M., RudnikA., JarząbM. et al., (2011): Epidermal growth factor receptor gene expression in high grade Gliomas. Folia Neuropathol; 49 (1): 28-38.

37. Puputti M., Tynninen O., Sihto H., Blom T., Mäenpa H., Isola J., Paetau A., Joensuu H. and Nupponen N. (2006): Amplification of KIT, PDGFRA, VEGFR2 , and EGFR in Gliomas. Mol Cancer Res; 4(12):927-34.

38. Avnider S., Sharma M. C., Deb P. et al., (2006) : Gemistocytic astrocytomas: histomorphology, proliferative potential and genetic alterations - a study of 32 cases. J Neurooncol; 78:123-7.

39. Alderson L. M., Castleberg R.L. and Harsh G. R. (1995): Human gliomas with wild type p53 express bcl2. Cancer Res; 55: 999-1001.

40. Rodriguez F. J. , Scheithauer B. W., Burger P. C., Jenkins S. and Giannini C. (2010): Anaplasia in Pilocytic Astrocytoma Predicts Aggressive Behavior. Am J Surg Pathol; 34:147-60.

41. Newcomb E.W., Sandhya K., Parrish C. L., Hayes R. L. Cohen H. and Miller D.C. (1997) : Bcl2 expression in astrocytoma in relation to patients survival and p53 gene status . Acta Neuropathologica; 94:369-75.

42. Rieger L., Weller M., Bornemam A. et al., (1998): Bcl2 family protein expression in human malignant glioma: a clinical pathological correlative study. J Neurol. Sci ; 155: 68-75.

43. Martin S., Toquet C., Oliver L. and Cartron P.F. et al.,(2001): Expression Of Bcl-2, Bax and Bcl-xl in Human Gliomas: A Re-appraisal . Neuro oncology J; 52: $129-39$.

44. Hussein M . R., Ghorori R. M. H. and Abd ElRahman Y. G. (2006): Alterations of p53, BCL-2, and $\mathrm{hMSH} 2$ protein expression in the normal brain tissues, gliosis, and gliomas. Int J Exp Pathol ; 87(4): 297-306.

45. Dietzmann K., von Bossanyi P., Sallaba J., Kirches E., Synowitz H.J. and Warich-Kirches M. (1996) : Immunohistochemically detectable p53 and mdm-2 oncoprotein expression in astrocytic gliomas and their correlation to cell proliferation. Gen Diagn Pathol. ;141(5-6):339-44.

46. Fels C., Schäfer C., Hüppe B., Bahn H., Heidecke V., Kramm C.M., Lautenschläger C. and Rainov N.G.(2000): Bcl-2 expression in higher-grade human glioma: a clinical and experimental study. J Neurooncol. ; 48(3):207-16. 